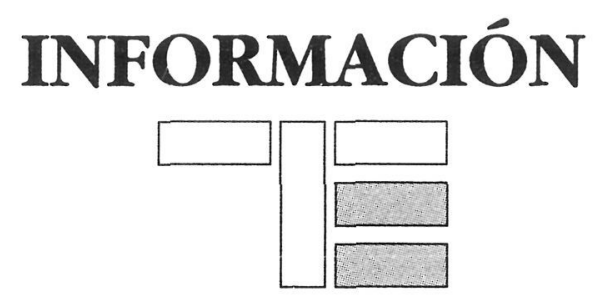




\section{Congreso Nacional de Teoría de la Educación}

Se ha celebrado en el Palacio de Congresos "Casa Colón" de Huelva los días 21, 22 y 23 de marzo de 2001 el VIII Congreso Nacional de Teoría de la Educación, que en esta edición ha abordado como tema de estudio la "Evaluación de las políticas educativas". Organizado por el Seminario Interuniversitario de Teoría de la Educación y la Universidad de Huelva, el congreso ha contado para esta ocasión con la colaboración de la Organización de Estados Iberoamericanos para la Educación, la Ciencia y la Cultura (OEI) y el Programa Cumbre de Cooperación Iberoamericana "Evaluación de la calidad de la educación". Durante tres días se ha analizado en qué medida la política educativa, en general, y las políticas educativas sectoriales están dirigiendo sus proyectos, tanto a nivel ideológico como económico, en una línea adecuada y pertinente a las necesidades reales que tienen los diferentes usuarios educativos y cómo están respondiendo los programas y acciones que se están aplicando en el ámbito educativo a las exigencias de calidad, eficacia y pertinencia que se reclama en la actualidad a los sistemas educativos.

Las sesiones de trabajo se organizaron en torno a cuatro ponencias que fueron impartidas por D. Julio Vera Vila (Universidad de Málaga), D. Joaquín García Carrasco (Universidad de Salamanca), D. a Sylvia Schemelkes del Valle (Secretaría de Educación Pública de México) y D. Jacques Hallak (Asesor Internacional de Educación de Unesco), que analizaron, respectivamente, cuestiones referidas a la evaluación de los profesores, las políticas de educación de adultos en la sociedad de la información, el papel de las organizaciones internacionales y las políticas de evaluación que vienen desarrollando y la educación y los derechos humanos en el contexto de la globalización. Asimismo, se estructuraron tres mesas redondas a cargo de investigadores y expertos de distintas administraciones autonómicas que giraron en torno a la evaluación de las políticas escolares, la cooperación internacional y la evaluación de la educación en Iberoamérica, así como una tercera mesa centrada en la sociedad civil y la evaluación de programas socio-educativos. 


\section{SEMINARIO INTERUNIVERSITARIO DE TEORÍA DE LA EDUCACIÓN. EDUCACIÓN Y CALIDAD DE VIDA.}

San Lorenzo de El Escorial - Noviembre de 2000

Continuando una tradición de casi 20 años, del 13 al 15 de noviembre de 2000 se celebra, en el Euroforum Felipe II del Real Sitio de San Lorenzo de El Escorial, el XIX Seminario Interuniversitario de Teoría de la Educación "Educación y calidad de vida", organizado por el Departamento de Teoría e Historia de la Educación y el Instituto de Ciencias de la Educación de la Universidad Complutense de Madrid, con la colaboración del Vicerrectorado de Investigación de esta universidad y el Ministerio de Ciencia y Tecnología.

Según la metodología de trabajo consolidada en estas reuniones, celebradas ininterrumpidamente desde 1982 en diferentes universidades españolas, el seminario se desarrolla a través de la discusión de cuatro ponencias marco, en las que colaboran 14 ponentes y una treintena de contribuciones en forma de addenda. Participan un total de 50 profesores de 22 universidades. Toda la documentación se distribuye para su examen con la suficiente antelación a través de la página de Internet del seminario. A solicitud de los asistentes, esta página queda como entorno permanente de información acerca de las actividades del SITE (www.ucm.es/info/site). La documentación es recogida igualmente en un CD editado por la organización del seminario, que facilita su consulta posterior. Los textos finales de las ponencias, revisados tras las reuniones del seminario, se publican en formato impreso en la Editorial Complutense. 